\title{
Entwarnung
}

\section{Saure Früchte begünstigen keine Inkontinenz}

— Zitrusfrüchten und Tomaten wird nachgesagt, dass sie die Harnblase irritieren und dadurch die Entstehung von Inkontinenz begünstigen. Für diese Behauptung lässt sich jedoch in einer großen prospektiven Studie keinerlei Beleg finden [Townsend MK et al. Int Urogyne-

col J 2012; doi: 10.1007/s00192-012-1914-9].

In der Subanalyse der Nurses' Health Stu-

dy (NHS) waren 65.168 Frauen zwi-

schen 37 und 79 Jahren, die zunächst

kontinent waren, zu ihren

Ernährungsgewohnheiten befragt und dann vier Jahre lang beobachtet worden. Selbst diejenigen Frauen, welche die meisten sauren Früchte und Frucht-

Zu Unrecht verdächtigt: Früchte mit hohem Säuregehalt wie Limetten
Frauen mit dem niedrigsten Konsum (eine Portion proTag). An diesem Null-Ergebnis änderte sich auch nichts, wenn man das Auftreten von Drang-, Belastungs- und geringfügiger Harninkontinenz einzeln betrachtete.

Ebenso wenig wird durch saure Früchte eine bestehende Harninkontinenz verschlechtert, wie die Überwachung von 23.063 bereits erkrankten NHS-Teilnehmerinnen zeigte. Eine Verschlimmerung trat innerhalb von zwei Jahren bei den Frauen mit dem höchsten und dem niedrigsten Konsum mit der gleichen Häufigkeit auf.

Unter dem Begriff ",saure Früchte“ wurden in der vorliegenden Studie Zitrusfrüchte, Pflaumen, Trauben, Äpfel, Beeren sowie Tomaten und Tomatenprodukte zusammengefasst. Auch bei getrennter Auswertung für die einzelnen Fruchtsorten, etwa für Zitrusfrüchte und Tomaten, ergaben sich keine Hinweise auf einen Zusammenhang mit der Entwicklung oder dem Fortschreiten einer Harninkontinenz. "Die Ergebnisse liefern erstmals eine epidemiologische Evidenz dafür, dass Frauen, die in Sorge sind wegen Inkontinenz, deswegen nicht den Verzehr von sauren Früchten beschränken müssen", schreiben die Studienautoren. Die Mediziner nennen auch einen möglichen Grund für die fehlende Korrelation: Die meisten säurehaltigen Früchte würden wegen ihres Gehalts an Karbonaten, Natrium und Kalium den Urin nämlich gar nicht sauer machen, sondern den pH-Wert sogar anheben.

Dr. Beate Schumacher

\section{OLG-Urteil}

\section{Fortbildung muss möglichst aktuell sein}

— Ärzte in Klinik und Praxis müssen sich regelmäßig fortbilden und auch gesicherte Erkenntnisse aus der einschlägigen Fachliteratur zeitnah umsetzen können. Nach einem Urteil des Oberlandesgerichts (OLG) Koblenz drohen ihnen sonst Schadenersatz- und Schmerzensgeldforderungen wegen grober Behandlungsfehler [Az.: 5 U 1450/11]. Geklagt hatte eine Patientin, die vor einer Operation die behandelnden Ärzte darauf hingewiesen hatte, dass sie die üblichen Narkosemittel nicht verträgt. Nach der Intubationsnarkose litt sie mehrere Tage an heftiger Übelkeit. Dies hätte mit der Gabe eines weiteren Medikaments, über das bereits ein Jahr vor dem Eingriff in der Fachliteratur berichtet wurde, jedoch verhindert werden können, so die Richter. Martin Wortmann

\section{Belastendes Erbe}

\section{„Brustkrebs-Gen“ erhöht Prostatakrebs-Risiko}

- Bekanntlich entwickeln Frauen mit BRCA1- oder BRCA2-Mutation häufiger Brust- und Eierstockkrebs. Aus epidemiologischen Studien mit Brustkrebsfamilien stammen daher auch die ersten Hinweise auf einen Zusammenhang zwischen diesen Mutationen und dem Auftreten von Prostatakrebs. Inzwischen ist für männliche BRCA2-Träger ein Anstieg des Prostatakarzinom-Risikos um mehr als das Achtfache gezeigt worden [Kote-Jarai Z et al. Br J Cancer 2011; 105: 1230-4]. Die gleiche britische Forschergruppe hat jetzt den Einfluss von BRCA-1-Mutationen untersucht [Leongamornlert $\mathrm{D}$ et al. Br J Cancer 2012; 106: 1697-701]. Dazu werteten sie die Sequenzdaten von 886 Prostatakrebspatienten aus.

Bei vier Patienten $(0,45 \%)$ wurden schädliche BRCA1-Mutationen nachgewiesen, die auch aus dem Brustkrebs-Register BIC bekannt waren. Bei den mit BRCA1 (und BRCA 2) assoziierten Prostatakarzinomen handelt es sich um besonders aggressive Tumoren. Etwa einer von 1.000 Männern, so eine Schätzung für Großbritannien, ist Träger einer BRCA1-Mutation. Unter Prostatakarzinompatienten sind der Studie zufolge knapp fünf von 1.000 betroffen. Das würde bedeuten, dass die Mutation das Prostatakrebsrisiko um den Faktor 3,75 vergrößert. Männer um die 65 mit einer BRCA1-Mutation hätten damit ein Erkrankungsrisiko von 8,6\%.

Zur Bestätigung ihrer Befunde empfehlen die Autoren, Männer, die schon in jungen Jahren an Prostatakrebs erkranken, routinemäßig auf BRCA1-Mutationen zu screenen. Wenn sich der Zusammenhang bestätige, könnte dies auch neue zielgerichtete Behandlungsoptionen eröffnen.

Dr. Beate Schumacher 